\title{
Inclusion of the Occupational Stress Model in Human Reliability Analysis
}

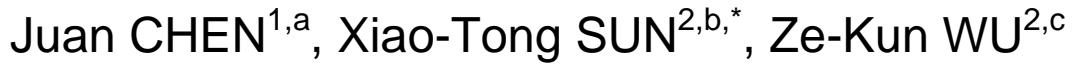 \\ ${ }^{1}$ Jimei University, Jimei District, Xiamen, Fujian, China \\ ${ }^{2}$ Beihang University, Xueyuan Road, \\ Haidian District, Beijing, China \\ ${ }^{3}$ Beihang University, Xueyuan Road, \\ Haidian District, Beijing, China \\ ajchen1979@163.com, bxttbb@buaa.edu.cn, cwuzekun@buaa.edu.cn
}

Keywords: HRA, PSFs, Occupational stress model, Yerkes-Dodson law.

\begin{abstract}
This study attempts to analysis human error from psychological perspective with introducing the occupational stress models into human reliability analysis (HRA). With classifying the performance shaping factors (PSFs) into two categories, we put forward the task demands and resources method. This method describes the qualitative relationship between the PSFs in the task context and the psychological processes of the operator. A human reliability experiment has been conducted and the experiment results proved the validity and flexibility in this method.
\end{abstract}

\section{Introduction}

As the system complexity increases in different industrial fields, the human-machine interactions have become more frequent. Thus, the accidents led by human factors have been gradually increased in recent years. According to the relative studies [1], human factors can be account for $60 \%$ of industrial accidents. In response to this situation, researchers have conducted a series of studies aiming at reducing human errors in the industrial field and the HRA is the main focus among all these efforts [2, 3]. Nevertheless, the existing HRA methods are tended to build direct relationship between the human performance and PSFs, which are influencing factors in the task context, while failing to consider the psychological process under such relationship.

In this paper, we incorporate the occupational stress model into the HRA and developed a complete process to analysis human error from psychological perspective. Firstly, we classify the PSFs into two types: the task demands and the task resources. Then, a qualitative approach is introduced to build the relationship between the two types of PSFs and the two crucial psychological factors: the motivation and the strain. After that, we go further to explore the human performance with different motivation intensities and stress level. An experiment has been conducted to verify our hypothesis and the experiment results proved the validity and flexibility in the task demands and resources model.

\section{Task Demands and Resources Method}

\section{Occupational Stress Model}

The occupational stress comes from the workers' interactions with the working environment and the factors that led to such stress are called "psychological stressors" [4]. The typical stressors are excessive time pressure, poor prospects for promotion, lack of work supporting, etc. The occupational stress models are designed to analyze the stressors' influences on workers' performance. There are two important characteristics in the occupational stress studies: firstly, the occupational stress model is capable of identifying and analyzing the stressors in the working environment; secondly, the occupational stress model is dedicated to improve the human performance from both physical and psychological aspects. We believe that these two important characteristics offer a validate basis for us to incorporate the existing occupational stress models into the HRA. With reference to the occupational stress model [5], we propose a new approach to analysis human error in the next session. 


\section{The Task Demands and Resources Model}

As indicated in the Fig.1, the task demands and resources model is a three-stages HRA method. It starts with identifying and classifying PSFs based on their potential influences on operator's psychological conditions. Then, the model conducts qualitative analysis on the relationship between the PSFs and the psychological factors of operator. Finally, the human error is determined with the operators' two crucial psychological factors: the motivation and the strain.

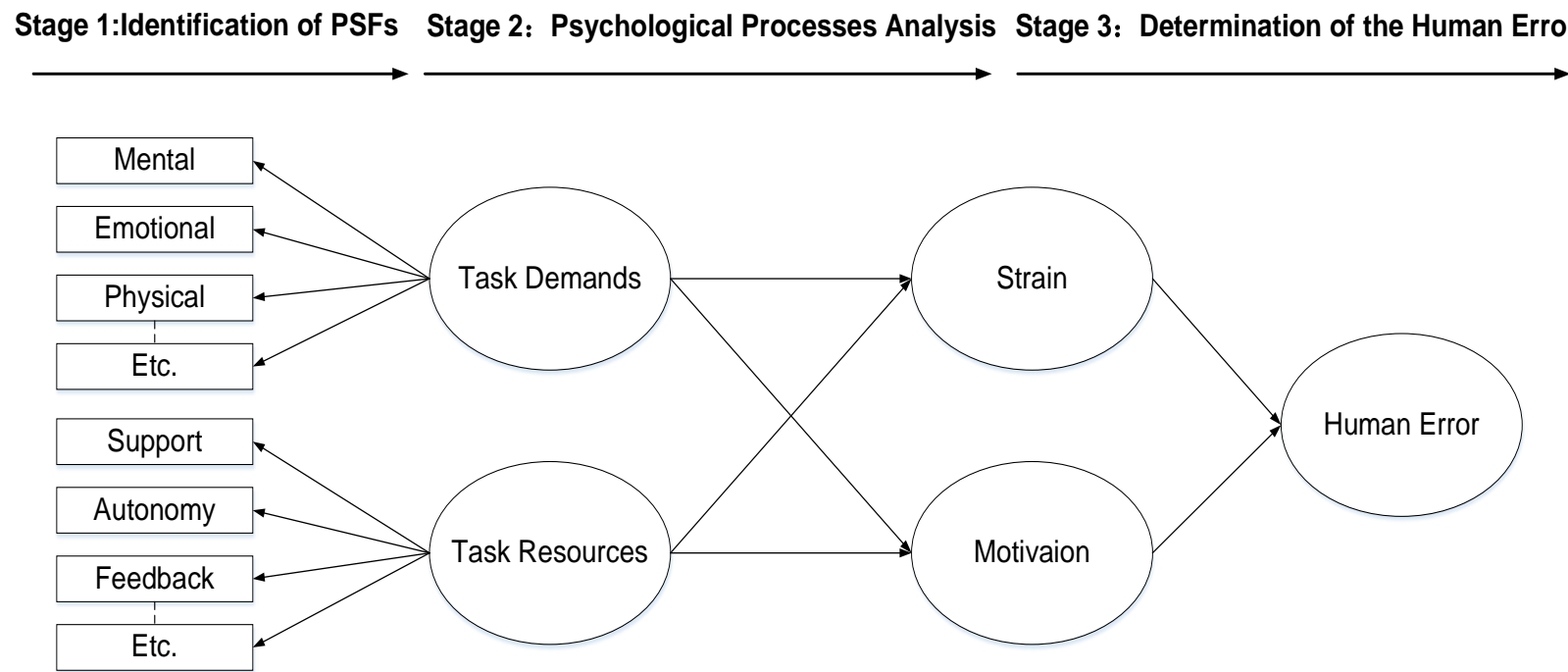

Fig.1. The Conceptual Model of the Task Demands and Resources Method

\section{Relationship between the Factors in the Model}

In this model, the task demands would increase the operator's subjective efforts and such PSFs would exhaust the operator from both mental and physical aspects [6]. In contrary, the task resources play as a significant role of fostering the operator's performance with motivating the operator and relieve the strain in the task [7].

Actually, there is no consensus on how motivation influences human performance. In this study, with reference to the Yerkes-Dodson Law [8], we assume that there would be an optimal motivation intensity for the operator in a specific task. As it comes to the effects of strain, there has been a series of discussions [9-11]. In 1985, Jamal tested all the main hypothesis with respect to the stress and human performance and the results supported a negative relationship between them [12].

\section{Experiment Research}

In order to verify the validity and flexibility of the task demands and resource model, we have conducted a human reliability experiment.

\section{Experiment Environment}

The experiment environment is a simulator based on a single-chip microcomputer. The simulator consists of a set of PAPI lamps, an LCD display, an inputting panel, an information processing system, and multiple controllers. In the experiment, guided by the indication of PAPI and the specific information on the display screen, the participants need to make corresponding operations as moving the throttles, adjusting the controllers and pushing the rudder button. The simulator would record all the participants' operation and produce an evaluation on their performances.

\section{Task Demands and Resources in the Experiment}

The first stage of the task demands and resource method is identifying the PSFs and categorizing them based on their influences on the operators' psychological conditions. We have chosen 8 PSFs that closely related to experimental simulation tasks and divided them into the task demands (task 
complexity, time pressure, unfavorable task schedule, unfavorable task conditions) and the task resources (economic compensation, performance feedback, support and training).

\section{Stress Level, Motivation Intensity and Human Error}

In the experiment, 60 healthy participants (36 men and 24 women) have been assigned into five different groups and the experiment condition for each group is corresponds to the task demands and resources that the participants received. Also, the stress level for different groups can be quantified based on expert evaluations on the different demands and resources in the task. To be more specific, the stress level $\mathrm{s}$ is estimated with the following equation:

$$
\mathrm{s}=\frac{1}{2}\left[\frac{\sum \mathrm{TD}}{\max \left(\sum \mathrm{TD}\right)}-\frac{\sum \mathrm{TS}}{\max \left(\sum \mathrm{TS}\right)}\right]+\frac{1}{2}
$$

where $\mathrm{s}(0<\mathrm{s}<1)$ is the estimated value for the stress level that based on the PSFs; $\sum$ TD is the number of PSFs in task demands that have a significant effects on the participants; $\sum$ TS is the number of PSFs in the task resources that have a significant effects on the participants; $\max \left(\sum \mathrm{TD}\right)$ and $\max \left(\sum \mathrm{TS}\right)$ are the maximum values of $\sum \mathrm{TD}$ and $\sum \mathrm{TS}$ respectively; $\Sigma / \max (\Sigma)$ is the normalized value of $\Sigma$.

Table 1. The evaluation of PSFs' effect on strain for group 1

\begin{tabular}{|c|c|c|}
\hline \multirow[t]{2}{*}{ PSFs } & \multicolumn{2}{|l|}{ Evaluation } \\
\hline & Experiment conditions & Effect on strain \\
\hline \multicolumn{3}{|c|}{ Task demands } \\
\hline Complexity & $\begin{array}{l}\text { The participants were asked to perform the task } \\
\text { as fast and as accurately as possible, which made } \\
\text { them face two simultaneous goals }\end{array}$ & S \\
\hline Time Pressure & $\begin{array}{l}\text { The available time for the task is set as } 6 \mathrm{~s} \text {, } \\
\text { comparing with the average time, } 9 \text { s, to complete } \\
\text { the task, the participants were under high time } \\
\text { pressure }\end{array}$ & S \\
\hline $\begin{array}{c}\text { Unfavourable task } \\
\text { schedule }\end{array}$ & $\begin{array}{l}\text { The experiment was conducted from 10:30 p.m. } \\
\text { to 11:00 p.m. and the participants' circadian } \\
\text { rhythms were considered in bad conditions }\end{array}$ & S \\
\hline $\begin{array}{l}\text { Unfavourable task } \\
\text { conditions }\end{array}$ & $\begin{array}{l}\text { The participants performed the whole task under } \\
\text { a dim lighting condition }\end{array}$ & S \\
\hline \multicolumn{3}{|c|}{ Task resources } \\
\hline $\begin{array}{l}\text { Economic } \\
\text { compensation }\end{array}$ & $\begin{array}{l}\text { The participants received economic } \\
\text { compensation from the experiment }\end{array}$ & S \\
\hline $\begin{array}{c}\text { Performance } \\
\text { feedback }\end{array}$ & $\begin{array}{l}\text { The participants were provided with feedback on } \\
\text { their performance in the task. }\end{array}$ & S \\
\hline Support & $\begin{array}{l}\text { The participants had to do the task on their own } \\
\text { and there was no support from any other during } \\
\text { the whole task }\end{array}$ & NS \\
\hline Training & $\begin{array}{l}\text { Besides the basic introduction to the experiment, } \\
\text { the participants did not receive any training for } \\
\text { the task. }\end{array}$ & NS \\
\hline
\end{tabular}

Note: S, significant, NS, not significant 
As indicated in the Tab. 1, the stress level for group 1 can be determined by the number of PSFs that have significant effects on the participants' psychological conditions. In this case, with the evaluation on the experiment conditions in group 1, the number of significant PSFs in task demands ( $\sum$ TD ) is 4. Similarly, the $\sum$ TS equals to 2. There are 4 total PSFs in task demands and resources separately $\left(\max \left(\sum\right)=4\right)$. With Eq. (1), the stress level for the group 1 can be calculated as $\mathrm{s}=\frac{1}{2}\left[\frac{4}{4}-\frac{2}{4}\right]+\frac{1}{2}=0.75$. Similarly, the stress levels for all the groups can be obtained as shown in Tab. 2 :

Table 2. The stress levels for the five experiment groups

\begin{tabular}{|c|c|c|c|c|c|}
\hline Group & $\mathbf{1}$ & $\mathbf{2}$ & $\mathbf{3}$ & $\mathbf{4}$ & $\mathbf{5}$ \\
\hline $\mathbf{s}$ & 0.75 & 0.5 & 0.375 & 0.25 & 0.125 \\
\hline
\end{tabular}

Compared with the stress level, the motivational intensity is more likely to vary with different individuals. Thus, it would be inappropriate to adopt expert evaluations to determine this psychological factor. In this experiment, we introduced a psychological scale to measure the motivational intensity for all participants. At the very beginning of the experiment, the participant was required to complete a questionnaire, which was adapted from the Situation Motivation Scale [13]. The questionnaire measures the motivation from four different dimensions and the motivation intensity can be quantified with the scores that the participants achieved in the questionnaire.

The quantification of human error is one of the key steps in all HRA methods. Referring to Swain and Guttman's definition of human error [2], the human error in the experiment was defined as the failure to complete the required task within a given time. Inspired by this definition, we chose the time pressure, which is one of the experiment conditions, as the exact criterion for the task being success or not. If the subject fails to complete the task within a certain level of time pressure, which corresponds to the available time in the task, the task would be considered as failed. Under this criterion, the human error can be quantified as a probability, which has an exact definition as the human error probability (HEP) in HRA [2]. In the experiment, the HEP can be calculated as a ratio between the number of failures in the experiment to the total number of task operations, as indicated in the following equation:

$$
\operatorname{HEP}=\frac{\sum_{\mathrm{i}=1}^{\mathrm{N}} \mathrm{f}\left(\mathrm{T}_{\mathrm{i}}\right)}{\mathrm{N}}, \mathrm{f}\left(\mathrm{T}_{\mathrm{i}}\right)= \begin{cases}1 & \mathrm{~T}_{\mathrm{i}}>\mathrm{T}_{\mathrm{a}} \\ 0 & \mathrm{~T}_{\mathrm{i}} \leq \mathrm{T}_{\mathrm{a}}\end{cases}
$$

where $T_{i}$ is the time taken by the participant to complete the task; $T_{a}$ is the available time to complete the task; $\mathrm{N}$ is the total times of task repetitions and is set as 50 in the experiment.

\section{Experiment Results}

In the Fig. 2, we present the scatter plots to display the participants' performance in different experimental groups. The $\mathrm{x}$-axis and the $\mathrm{y}$-axis represents the motivational intensity and HEP separately. Each point contains the information of the participant' motivation intensity and HEP under a specific stress level. As in indicated in the Fig. 3, the group 1 was under the highest stress level(s=0.75) and the participants in this group reached relative higher HEP among all the groups. In contrast, participants in group 5 , which was under the lowest stress level ( $\mathrm{s}=0.125)$, achieved lower HEP. Intuitively, the HEP rises with the stress levels and the participants' performances differs with the task demands and resources they received. To verify this point, we have performed an ANOVA test on the HEP in different groups and it turned out that the stress level, which corresponds the different task demands and resources, did have a significant influence on HEP $(p<0.05)$. 


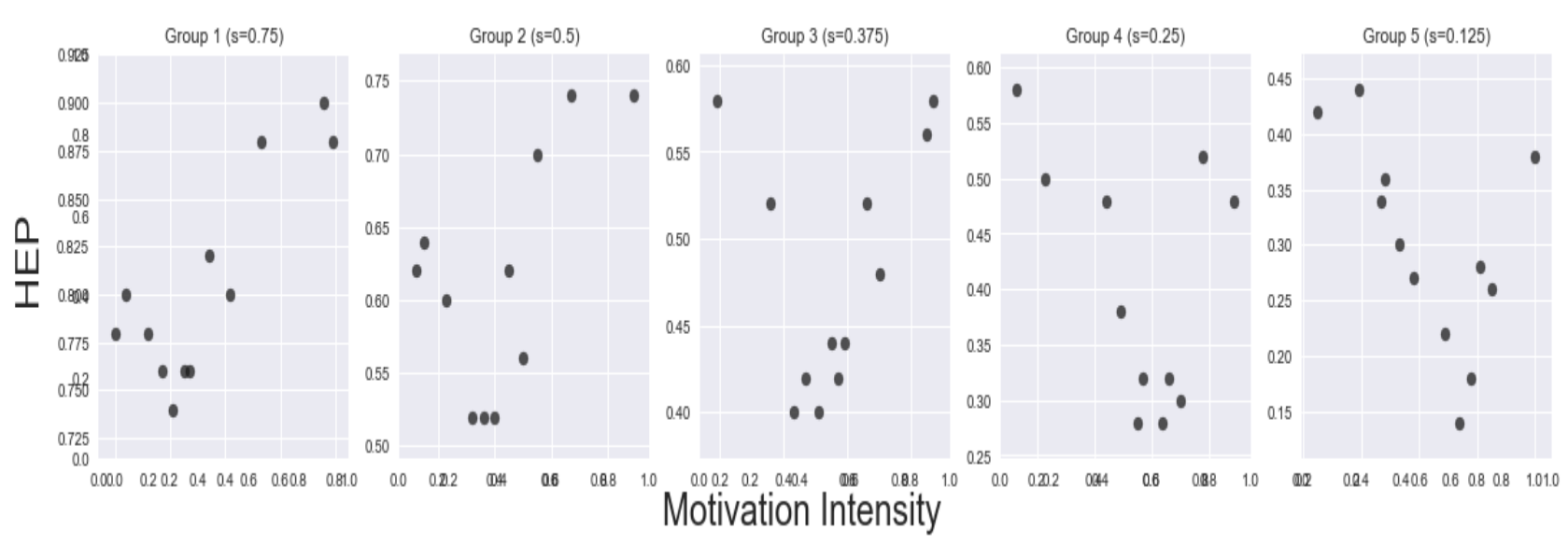

Fig. 2. The Scatter Plots for the Experiment Results

Another crucial characteristic that we can observe in the experiment results is the U-shape trend in the way that the HEP goes with the motivation intensity. Although it would be impractical to quantify the HEP with the actual motivation in a complicated task context, such qualitative relationship reveals the possible existence of the optimal motivation for an operator in a specific task.

\section{Conclusion}

In this paper, we include the occupational stress model into the human reliability analysis and produce a new approach as the task demands and resources method. This method is focused on the operator's psychological process in a specific task and determine the human error with a three-stages analysis process. To verify this model, we conducted the human reliability experiment and the experimental results support our hypothesis about the qualitative relationships between the motivation, strain and human performance.

\section{References}

[1] Griffith, C.D. and S. Mahadevan, Inclusion of fatigue effects in human reliability analysis. Reliability Engineering \& System Safety, 2011. 96(11): p. 1437-1447.

[2] Swain, A. and H. Guttman, Handbook of human reliability analysis with emphasis on nuclear power plant applications. Technical Report, 1983.

[3] Hollnagel, E., - Cognitive Reliability and Error Analysis Method (CREAM). Cognitive Reliability \& Error Analysis Method, 1998: p. 262-275.

[4] Lewin, K., Field theory in social science: selected theoretical papers (Edited by Dorwin Cartwright.). Psychological Bulletin, 1951. 48(6): p. 520-521.

[5] Demerouti, E., et al., The Job Demands: Resource Model of Burnout. Journal of Applied Psychology, 2001. 86(3): p. 499-512.

[6] Meijman, T.F. and G. Mulder, Psychological Aspects of Workload. New Handbook of Work \& Organizational Psychology, 1998.

[7] Hobfoll, S.E., The Influence of Culture, Community, and the Nested - Self in the Stress Process: Advancing Conservation of Resources Theory. Applied Psychology, 2001. 50(3): p. 337-421.

[8] Teigen, K.H., Yerkes-Dodson: A law for all seasons. Theory \& Psychology, 1994. 4(4): p. 525-547. 
[10] Gupta, N. and T.A. Beehr, Job stress and employee behaviors. Organizational Behavior \& Human Performance, 1979. 23(3): p. 373-387.

[11] Barsky, A., et al., Modeling negative affectivity and job stress: a contingency-based approach. Journal of Organizational Behavior, 2004. 25(8): p. 915-936.

[12] Jamal, M., Relationship of Job Stress to Job Performance: A Study of Managers and Blue-Collar Workers. Human Relations, 1985. 38(5): p. 409-424.

[13] Standage, M., et al., Validity, reliability, and invariance of the Situational Motivation Scale (SIMS) across diverse physical activity contexts. Journal of Sport \& Exercise Psychology, 2003. 25(1): p. 19-43. 